\title{
ASOCIACIÓN DEL ÍNDICE DE MASA CORPORAL Y EL AUTOCONTROL CON LA CALIDAD DE VIDA RELACIONADA CON LA SALUD EN PERSONAS CON SOBREPESO/OBESIDAD*
}

\author{
ASSOCIATION OF BODY MASS INDEX AND SELF-CONTROL WITH HEALTH- \\ RELATED QUALITY OF LIFE IN OVERWEIGHT/OBESE PEOPLE
}

\begin{abstract}
MONSERRAT MEDRANO-VÁZQUEZ ${ }^{1 * * *}$, MARIO E. ROJAS-RUSSELL ${ }^{1,2, * * * *}$, KARINA SERRANO-ALVARADO $^{1 * * * * *}$, LUIS $^{2}$ FLÓREZ-ALARCÓN ${ }^{3 * * * * *}$, ÁNGELES AEDO-SANTOS ${ }^{2 * * * * *}$, MALAQUÍAS LÓPEZ-CERVANTES $^{2 * * * * * * *}$

${ }^{1}$ Universidad Nacional Autónoma de MÉXico, Facultad de ESTUdios SUPERIORES Zaragoza, MÉXico D. F. - MÉXico

${ }^{2}$ Universidad Nacional AutónOma de MÉXico, FACULTAd de Medicina, MÉXico D. F. - MÉXico

${ }^{3}$ UnIVERSIDAD NACIONAL DE COLOMBIA, BOGOTÁ - COLOMBIA
\end{abstract}

FECHA RECEPCIÓN: 20/11/2013・ FECHA ACEPTACIÓN: 29/12/2013

Para citar este artículo: Medrano-Vázquez, M., Rojas-Russell, E., Serrano-Alvarado, K., Flórez-Alarcón, L., Aedo-Santos, A., \& López-Cervantes, M. (2014). Asociación del índice de masa corporal y el autocontrol con la calidad de vida relacionada con la salud en personas con sobrepeso/obesidad. Psychologia: avances de la disciplina, 8(1), 13-22

\begin{abstract}
Resumen
El propósito de este trabajo fue identificar la asociación del autocontrol con la calidad de vida relacionada con la salud y el papel que en ella pudiera tener el Índice de Masa Corporal (IMC). Se realizó un estudio transversal predictivo. Participaron 273 adultos, usuarios de un centro de primer nivel de atención a la salud de la Ciudad de México, entre 18 y 65 años de edad, con un IMC mayor a 25 unidades. Se aplicó el SF12 v1 y la escala de autocontrol de Tangney, Baumeister y Boone. Se identificó una asociación significativa entre el autocontrol y la calidad de vida física y mental; el IMC se asoció con la calidad de vida física, pero no con la calidad de vida mental, ni con el autocontrol. No se identificó un efecto mediador del IMC en la relación del autocontrol con la calidad de vida. Los resultados sugieren que una persona con sobrepeso u obesidad con mayor autocontrol, tiene mejor percepción de bienestar en aspectos físicos y emocionales, independientemente del IMC.

Palabras clave: calidad de vida, índice de masa corporal, obesidad, centros de salud
\end{abstract}

\footnotetext{
* Financiamientos: PAPIIT-UNAM: Proyecto IN304812. CONACYT: Proyecto SALUD-2012-01-181118. Responsable: Dr. Mario E. Rojas-Russell

** Privada San Luis Potosí Mz 26, Lt 10, Ampliación San Agustín, Chimalhuacán, Estado de México, CP 56346, México. monserrat.medrano.v@ gmail.com

*** Arenal 694-F704, Col. Arenal-Tepepan, Tlalpan DF, CP 14610.merr@unam.mx

**** Tulipan 4, Col Loma Linda, Naucalpan de Juárez, Estado de México, CP 56318, México. karina_230282@hotmail.com

******Universidad Nacional de Colombia, Departamento de Psicología, Bogotá, Colombia. luis@florez.info.co

******* Paseo del Rio 68, Col, Chimalistac, D. F., México. aedoaa@gmail.com

******* Gama 125, Col. Rinconada San Jerónimo, Cuernavaca, Morelos. CP 62170, México. mlopez14@unam.mx
} 


\begin{abstract}
The aim of this study was to identify the association of self-control with health-related quality of life and the possible mediation of the Body Mass Index (BMI). A predictive cross-sectional study was conducted. Participated 273 adults, users of a primary health care center in Mexico City, aged 18 to 65 years, with a BMI greater than 25. The SF-12 V1 and the Tangney, Baumeister and Boone's Self-Control Scale were applied. The results showed a significant association between self-control to physical and mental health quality of life; BMI was associated with physical quality of life. No associations were found between BMI to mental quality of life and self-control. There were no evidence of a mediation effect of BMI on the relationship between self-control and health-related quality of life. The results suggest that an overweight or obese person with greater self-control has better perception of physical and emotional well-being, independently of BMI.

Keywords: quality of life, body mass index, obesity, health centers
\end{abstract}

\section{Introducción}

Existen diferentes modelos conceptuales que definen la Calidad de Vida Relacionada con la Salud (CVRS). Sin embargo, dos aspectos centrales integran la mayoría de las definiciones de CVRS: es subjetiva, es decir, debe ser evaluada desde la perspectiva del paciente siempre que sea posible; y es multidimensional: incluye múltiples facetas de la vida y la situación del paciente (Ruiz \& Pardo, 2005; Vinaccia \& Quiceno, 2012).

Para Torre et al., (2008) en la CVRS se diferencian dos dimensiones: una funcional, que incluye las actividades diarias, como el cuidado de uno mismo, los trabajos remunerados o no y las relaciones sociales con la familia o los amigos; y la otra subjetiva, que es el sentimiento de «cómo se encuentra uno mismo». Es decir, si la persona se siente feliz o triste, si se encuentra cansado o con mucha energía, si está con dolor o sin él.

Uno de los principales enfoques a los que se ha orientado la investigación de la CVRS es el que se refiere a la percepción que tiene el paciente de los efectos de una enfermedad determinada, sobre su calidad de vida en general (Romero-Márquez \& Romero-Zepeda, 2010).

En este sentido, el incremento del Índice de Masa Corporal (IMC) se ha asociado con la reducción de la CVRS (Jia \& Lubetkin, 2005; Kearns, Ara, Young, \& Relton, 2013). Algunos estudios apoyan la observación de que las personas obesas tienen una peor calidad de vida física en comparación con las personas con un peso normal (Takahashi et al., 2011). Sin embargo, la asociación no parece ser tan consistente con la calidad de vida mental (Renzaho, Wooden \& Houng, 2010).

La obesidad es consecuencia de un desbalance energético crónico relacionado casi siempre con factores modificables: el comportamiento alimentario, específicamente la ingesta calórica excesiva, el tamaño de las porciones, la accesibilidad de los alimentos y la actividad física (López-Villalta \& Soto, 2010).

Al respecto, un insuficiente autocontrol, entendido como la capacidad para modificar las propias respuestas y ponerlas en consonancia con las normas, los ideales, los valores, la moral, las expectativas sociales y la persecución de objetivos a largo plazo, se ha relacionado con problemas de comportamiento y control de los impulsos, incluyendo comer en exceso, el abuso del alcohol y las drogas, la delincuencia y la violencia, el gasto excesivo y el comportamiento sexual impulsivo, etc. (Baumeister, Vohs, \& Tice, 2007).

Sin embargo, actualmente es poco conocida la relación entre el autocontrol y los resultados en la salud física y mental (Boals, Vandellen \& Banks, 2011).

En un estudio, con adolescentes, se identificó que un bajo autocontrol se asoció con condiciones adversas de salud, lo que sugiere que aquél se asocia con resultados negativos en las dimensiones de bienestar físico y psicológico (Miller, Barnes \& Beaver, 2011).

Boals et al. (2011), encontraron que el autocontrol se relacionó con menores niveles de depresión, abuso de alcohol y percepción de estrés, y con mayores niveles de satisfacción con la vida. Además, sugieren que un menor autocontrol está asociado con la evitación como estilo de afrontamiento, y esto a su vez, con una precaria salud física y mental.

Aunque es poca, la evidencia muestra que un mayor autocontrol percibido parece afectar de manera positiva la valoración subjetiva de bienestar y la puesta en práctica de conductas de autocuidado relacionadas con resultados favorables a la salud, entre ellos el control del peso corporal, el cual al aumentar, afecta negativamente la percepción de CVRS.

Por todo lo anterior, el objetivo de este trabajo fue identificar la asociación del autocontrol con la calidad 
de vida relacionada con la salud y el papel que en ella pudiera tener el IMC en una muestra de usuarios con sobrepeso u obesidad de un centro de salud de primer nivel de atención. Este trabajo forma parte de un estudio más amplio sobre intervenciones motivacionales en personas con sobrepeso u obesidad. Los datos que se presentan corresponden a algunas de las variables evaluadas en la medición basal de ese estudio.

\section{Método}

\section{Participantes}

Se llevó a cabo un estudio transversal predictivo con usuarios de un centro de primer nivel de atención a la salud de la Ciudad de México, elegidos a través de un muestreo accidental. Se consideraron como criterios de inclusión personas entre 18 y 65 años de edad, con un IMC mayor a 25, quienes de forma voluntaria firmaron una carta de consentimiento informado; se excluyeron personas analfabetas y mujeres embarazadas. Se entrevistaron 290 participantes, de los cuales 8 no cumplieron con el rango de edad y 9 tenían un IMC menor a 25, por lo que la muestra final estuvo conformada por 273 participantes.

\section{Instrumentos}

Cuestionario de Salud SF-12 versión 1: Se trata de una versión reducida del Cuestionario de Salud SF-36. Se empleó la adaptación realizada en España, por Alonso (s. f.) del SF-12 Health Survey. Consta de 12 ítems provenientes de las 8 dimensiones del SF-36: Función Física (2 ítems), Función Social (1 ítem), Rol físico (2 ítems), Rol emocional (2 ítems), Salud mental (2 ítems), Vitalidad (1 ítem), Dolor corporal (1 ítem), Salud general (1 ítem). Las opciones de respuesta están en una escala de tipo Likert que evalúa la intensidad o frecuencia de la condición que se pregunta y que proporcionan un perfil de salud, que se resume en dos componentes; uno físico y otro mental. El índice de confiabilidad reportado con población española fue de .8 (Vilagut et al., 2008).

Autocontrol (Brief Self-control Scale, Tangney, Baumeister \& Boone, 2004): este instrumento evalúa el grado de autocontrol que presentan las personas en relación a cinco dominios: control de pensamientos, control de emociones, control de impulsos, regulación de la conducta y ruptura de hábitos. Es una escala compuesta por 13 ítems en formato tipo Likert, con un rango de respuesta entre 1 (totalmente en desacuerdo) y 5 (totalmente de acuerdo). El índice de confiabilidad reportado con población española fue de .82 (Oliva et al., 2012).

Índice de Masa Corporal (IMC): se calcula dividiendo el peso de una persona en kilos por el cuadrado de su talla en metros $\left(\mathrm{kg} / \mathrm{m}^{2}\right)$. Un IMC igual o superior a 25 determina sobrepeso, mientras que uno igual o superior a 30 determina obesidad (Organización Mundial de la Salud, 2011).

\section{Procedimiento}

Los participantes fueron reclutados en las salas de espera de un centro de salud de la Ciudad de México, donde se les explicó el propósito del estudio. Los participantes potenciales se encontraban en el centro de salud por motivos de consulta distintos al control de peso. Después de aceptar participar en el estudio, firmaron un consentimiento informado. Posteriormente se les entregaron las pruebas psicométricas que medían aspectos psicológicos relacionados con dieta, actividad física y salud general, entre ellos la escala de autocontrol y el SF-12. Finalmente, psicólogos previamente capacitados y estandarizados midieron la talla y el peso corporal, con un estadímetro portátil marca Seca modelo 213 y una báscula digital marca Tanita modelo UM061.

\section{Análisis de datos}

Se utilizó el paquete estadístico STATA v 12. Se realizaron análisis descriptivos, bivariados y un análisis de mediación con modelos de regresión lineal de acuerdo al modelo de Mckinnon (2008). Asimismo, se realizaron análisis de factor confirmatorio (AFC) y de consistencia interna (CI) para evaluar las propiedades psicométricas de los instrumentos empleados en este estudio.

\section{Resultados}

El número total de participantes en el estudio fue de 273 , de los cuales, el $86.5 \%$ fueron mujeres, con una edad promedio de 42 años $(\mathrm{SD}=11.8)$. El $26 \%$ de los participantes se ubicaron en la categoría sobrepeso y el $74 \%$ restante en obesidad. En la Tabla 1, se muestran las características de las variables sociodemográficas y antropométricas. 
Tabla 1. Características sociodemográficas y antropométricas

\begin{tabular}{|c|c|c|c|}
\hline $\begin{array}{l}\text { Variables } \\
\text { Sociodemográficas }\end{array}$ & $\begin{array}{l}\% \\
(n=273)\end{array}$ & $\begin{array}{l}\text { Variables } \\
\text { Antropométricas }\end{array}$ & $(n=260)$ \\
\hline Sexo & & Peso & \\
\hline Femenino & 86.45 & Media & 82.4 \\
\hline Masculino & 13.55 & Desviación estándar & 18.1 \\
\hline Estado civil & & Talla & \\
\hline Soltero & 24.26 & Media & 156.5 \\
\hline Casado/unión libre & 66.18 & Desviación estándar & 7.6 \\
\hline Otro & 9.56 & IMC & \\
\hline Ocupación & & Media & 33.5 \\
\hline Profesional & 4.80 & Desviación estándar & 6.0 \\
\hline Comerciante & 18.08 & & \\
\hline Trabajador por cuenta propia & 19.93 & & \\
\hline Ama de casa & 48.34 & & \\
\hline Estudiante & 3.32 & & \\
\hline Desempleado & 5.54 & & \\
\hline \multicolumn{4}{|l|}{ Escolaridad } \\
\hline Primaria o menos & 24.26 & & \\
\hline Secundaria & 31.25 & & \\
\hline Bachillerato o más & 44.49 & & \\
\hline \multicolumn{4}{|l|}{ Edad } \\
\hline Media & 41.70 & & \\
\hline Desviación estándar & 11.81 & & \\
\hline \multicolumn{4}{|l|}{ Nivel socioeconómico } \\
\hline Rango & $1-10$ & & \\
\hline Media & 4.57 & & \\
\hline Desviación estándar & 1.55 & & \\
\hline
\end{tabular}

Nota: En 13 casos no fue posible obtener el peso, la talla o ambos.

En la escala de autocontrol se presentaron $22 \mathrm{ca}-$ sos en los que los participantes omitieron una sola respuesta, en estos casos, para poder estimar el puntaje total de la escala se interpolaron los ítems faltantes, promediando las puntuaciones de los reactivos de cada uno de los participantes.

En el AFC de la escala de autocontrol, los índices de bondad de ajuste fueron: $\chi^{2}=45 ; \mathrm{p}=.31$; CFI 0.99; TLI .98; SRMR 0.04 y R $\mathrm{R}^{2} 0.71$; para el componente físico del SF-12,: $\chi^{2}=1.63 ; \mathrm{p}=.90$; CFI 1.00; TLI 1.02; SRMR 0.01, y R $\mathrm{R}^{2} .73$ y para el componente mental: $\chi^{2}=1.11 ; \mathrm{p}=.89$; CFI 1.00; TLI 1.02;
SRMR 0.00 y $\mathrm{R}^{2}$.80. En todos los casos los indicadores de bondad de ajuste se comportaron dentro de los parámetros deseables para este tipo de análisis (Ruiz, 2000).

Un primer análisis de CI de la escala de autocontrol, mostró un alfa de Cronbach igual a .64, el mismo análisis indicó que eliminando los reactivos 6,8 y 11 , el coeficiente de CI se incrementaba a .68, por tal razón, los análisis posteriores se realizaron sin los tres ítems mencionados. Para el SF-12 se realizó el análisis correspondiente por componente, el físico presentó un alfa de .80 y el mental de .76 . 
Se identificó el tipo de distribución de los datos. En el caso del IMC se transformó a su inverso (1/IMC) para normalizar la variable.

Se efectuó un análisis bivariado, con pruebas no paramétricas Kruskal-Wallis y U de MannWhitney, entre los componentes físico y mental de calidad de vida y variables sociodemográficas. La escolaridad mostró diferencias significativas en calidad de vida física $\left(\chi^{2}=11.68, \mathrm{p}<.01\right)$, las personas con una escolaridad de bachillerato o más, presentaron una mejor CVRS comparadas con las de primaria $(\mathrm{p}<.01)$ y secundaria $(\mathrm{p}<.01)$. Por su parte, el sexo presentó diferencias en la calidad de vida mental $(\mathrm{z}=2.57, \mathrm{p}<.01)$ y física $(\mathrm{z}=2.87$, $\mathrm{p}<.01$ ), en ambos casos los hombres tuvieron mayores puntuaciones.

Posteriormente, se realizó un análisis de mediación simple con ecuaciones de regresión lineal (MacKinnon, 2008) ajustado por sexo y escolaridad para determinar la posible asociación entre las variables de interés y el efecto mediador del IMC. Estos análisis se realizaron con los registros que tuvieran todos los datos en las variables de interés. En la Figura 1 se representa el modelo de mediación a prueba.

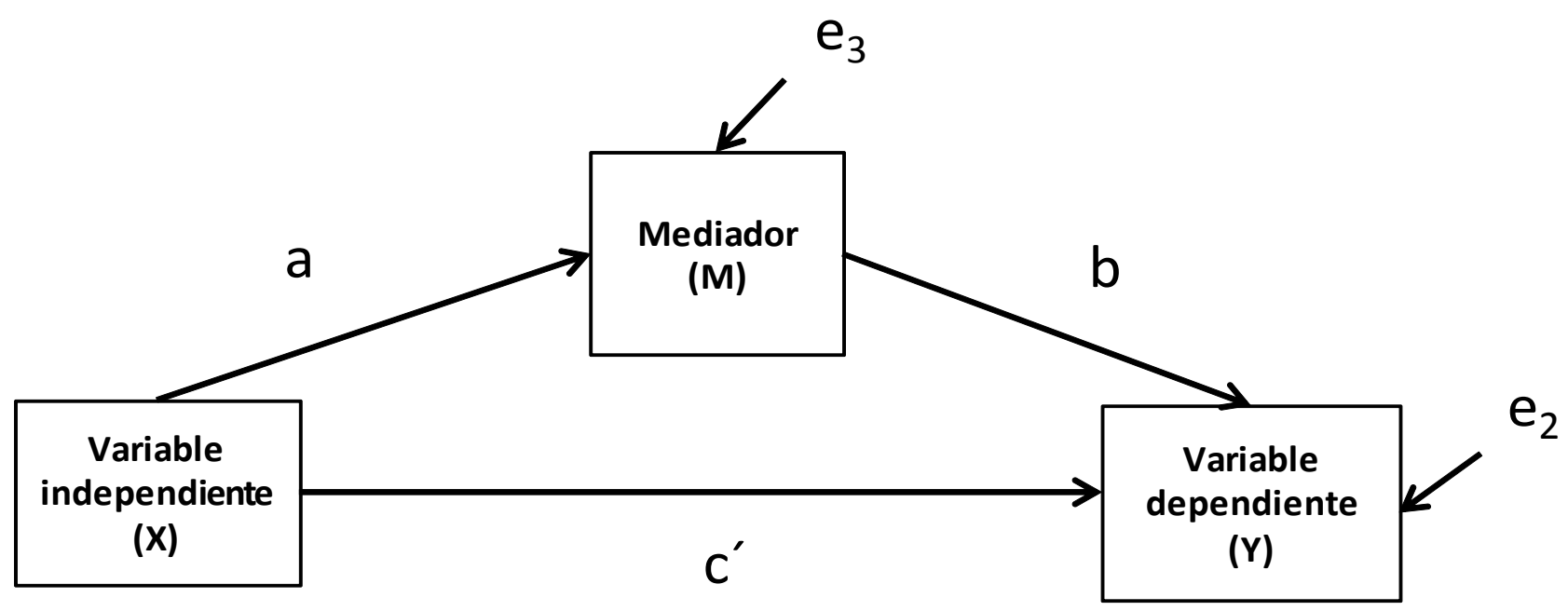

Figura 1. Modelo de mediación

Este modelo implica tres ecuaciones de regresión: la primera destinada a estimar la relación de $\mathrm{X}$ con Y (c); la segunda evalúa el efecto de X en Y después de ser ajustado por $M\left(c^{\prime}\right)$, además de la relación de $\mathrm{M}$ con $\mathrm{Y}$ ajustado por X (b); la tercera valora la asociación de M con X (a). El efecto mediador resulta del producto de $a$ y $b\left(a^{*} \mathrm{~b}\right)$ (MacKinnon, 2008).

La tabla 2 muestra los coeficientes de regresión estimados mediante el método robusto, para CVRS física, resultado de las tres ecuaciones de regresión implicadas en el modelo de mediación. Se empleó este método de estimación ya que permite relajar los supuestos de normalidad que exige el método de mínimos cuadrados ordinarios.

La CVRS física se asoció significativamente con el autocontrol, las mujeres presentan una menor
CVRS física comparadas con los hombres, también se observa que el tener mayor nivel académico se asocia con la variable dependiente, es decir las personas que tienen una escolaridad bachillerato o más tienen una mejor CVRS física comparadas con las de primaria o menos.

Estas mismas asociaciones se mantienen al ajustar por IMC; se presentó una asociación significativa entre CVRS física y el IMC. Debido a que la relación entre IMC y su inverso (1/IMC) es inversamente proporcional, la asociación se presenta en la dirección esperada, a mayor IMC menor CVRS física.

No se observó una asociación entre el IMC y el autocontrol, y por tanto, tampoco un efecto mediador del IMC en la asociación del autocontrol con la CVRS física. 
Tabla 2. Modelos de regresión lineal para el análisis de mediación del imc sobre la relación autocontrol y calidad de vida física.

\begin{tabular}{|c|c|c|c|c|c|c|}
\hline & Variable & Coeficiente & ES & $\mathrm{t}$ & IC 95\% & $\mathrm{R}^{2}$ \\
\hline Modelo $1(n=273)$ : & Autocontrol & $1.06 *$ & .17 & 6.3 & .71 .4 & .17 \\
\hline \multirow[t]{7}{*}{ CVRS Física } & Género & & & & & \\
\hline & Hombres & Referencia & & & & \\
\hline & Mujeres & $-9.9 *$ & 3.6 & -2.7 & $-16.9-2.8$ & \\
\hline & Escolaridad & & & & & \\
\hline & Primaria & Referencia & & & & \\
\hline & Secundaria & 3.5 & 3.6 & 0.98 & -3.610 .6 & \\
\hline & Bachillerato o más & $9.8 *$ & 3.5 & 2.8 & 2.816 .7 & \\
\hline Modelo $2(n=258)$ : & Autocontrol & $1.01 *$ & .17 & 5.8 & .71 .3 & .19 \\
\hline \multirow[t]{8}{*}{ CVRS Física } & $\mathrm{IMC}^{\#}$ & $867.8 *$ & 268.5 & 3.2 & 3391396.7 & \\
\hline & Género & & & & & \\
\hline & Hombres & Referencia & & & & \\
\hline & Mujeres & $-9.9 *$ & 3.7 & -2.7 & $-17.1-2.6$ & \\
\hline & Escolaridad & & & & & \\
\hline & Primaria & Referencia & & & & \\
\hline & Secundaria & 3.4 & 3.5 & 0.9 & -3.510 .4 & \\
\hline & Bachillerato o más & $8.7 *$ & 3.5 & 2.5 & 1.815 .6 & \\
\hline Modelo $3(n=258)$ : & Autocontrol & .00 & .00 & .8 & -.00 .00 & .01 \\
\hline \multirow[t]{7}{*}{$\mathrm{IMC}^{\#}$} & Género & & & & & \\
\hline & Hombres & Referencia & & & & \\
\hline & Mujeres & .00 & .00 & 1.3 & -.00 .00 & \\
\hline & Escolaridad & & & & & \\
\hline & Primaria & Referencia & & & & \\
\hline & Secundaria & .00 & .00 & 1.2 & -.00 .00 & \\
\hline & Bachillerato o más & .00 & .00 & .9 & -.00 .00 & \\
\hline
\end{tabular}

Nota. \#Inverso (1/IMC); * p < 0.05; ES: error estándar; IC 95\% intervalo de confianza al 95\%

La CVRS mental mostró una asociación positiva con el autocontrol; nuevamente las mujeres presentaron una menor CVRS mental comparadas con los hombres, en este caso la escolaridad no presentó una asociación significativa. Estas relaciones se mantuvieron aun estando ajustadas por el IMC (Tabla 3).

Por otro lado, el IMC no se asoció con la calidad de vida mental y tampoco con el autocontrol, por lo que se rechaza un posible efecto mediador. 
Tabla 3. Modelos de regresión lineal para el análisis de mediación del imc sobre la relación autocontrol y calidad de vida mental.

\begin{tabular}{|c|c|c|c|c|c|c|}
\hline & Variable & Coeficiente & ES & $\mathrm{t}$ & IC 95\% & $\mathrm{R}^{2}$ \\
\hline Modelo 1 $(n=273)$ : & Autocontrol & $1.2 *$ & .19 & 6.2 & .811 .6 & .14 \\
\hline \multirow[t]{5}{*}{ CVRS Mental } & $\begin{array}{l}\text { Género } \\
\text { Hombres }\end{array}$ & Referencia & & & & \\
\hline & Mujeres & $-9.6 *$ & 3.4 & -2.8 & $-16.3-2.9$ & \\
\hline & $\begin{array}{l}\text { Escolaridad } \\
\text { Primaria }\end{array}$ & Referencia & & & & \\
\hline & Secundaria & -2.5 & 4.1 & -0.6 & -10.55 .5 & \\
\hline & Bachillerato o más & -2.6 & 3.8 & -0.7 & -10.14 .9 & \\
\hline Modelo $2(n=258)$ : & Autocontrol & $1.2 *$ & .20 & 5.8 & .781 .6 & .14 \\
\hline \multirow[t]{6}{*}{ CVRS Mental } & $\mathrm{IMC}^{\#}$ & 175.6 & 302.1 & 0.6 & -419.4770 .5 & \\
\hline & $\begin{array}{l}\text { Género } \\
\text { Hombres }\end{array}$ & Referencia & & & & \\
\hline & Mujeres & $-10.7 *$ & 3.4 & -3.1 & $-17.4-3.9$ & \\
\hline & $\begin{array}{l}\text { Escolaridad } \\
\text { Primaria }\end{array}$ & Referencia & & & & \\
\hline & Secundaria & -2.2 & 4.2 & -0.5 & -10.46 .0 & \\
\hline & Bachillerato o más & -2.8 & 3.9 & -0.7 & -10.64 .9 & \\
\hline Modelo $3(n=258)$ : & Autocontrol & .00 & .00 & .8 & -.00 .00 & .01 \\
\hline \multirow[t]{5}{*}{$\mathrm{IMC}^{\#}$} & $\begin{array}{l}\text { Género } \\
\text { Hombres }\end{array}$ & Referencia & & & & \\
\hline & Mujeres & .00 & .00 & 1.3 & -.00 .00 & \\
\hline & $\begin{array}{l}\text { Escolaridad } \\
\text { Primaria }\end{array}$ & Referencia & & & & \\
\hline & Secundaria & .00 & .00 & 1.2 & -.00 .00 & \\
\hline & Bachillerato o más & .00 & .00 & .9 & -.00 .00 & \\
\hline
\end{tabular}

Nota: \# Inverso (1/IMC) ; *p < 0.05; ES: error estándar; IC 95\% intervalo de confianza al $95 \%$

\section{Discusión}

Se identificaron asociaciones positivas entre el autocontrol y los componentes físico y mental de la calidad de vida, esto sugiere que una persona con mayor autocontrol en áreas como el control de pensamientos, emociones e impulsos, además de la regulación de la conducta y ruptura de hábitos, tiene mejor percepción de bienestar en aspectos físicos y emocionales, relacionados con un adecuado desempeño en sus actividades cotidianas, en sus relaciones sociales y, en consecuencia, en su salud general. En este sentido, conductas en cuya realización está implicado el autocontrol, por el constante requerimiento de esfuerzo para adherirse a rutinas y/o manejar pensamientos y emociones, tales como la dieta y el ejercicio, se han asociado con una mejor calidad de vida (Hassan, Joshi, Madhavan \& Amonkar, 2003).

El IMC se asoció con la CVRS física, es decir, a mayor IMC peor calidad de vida, sin embargo, la asociación no se presentó para la CVRS mental. Hay evidencia que apoya 
esta diferencia, un estudio mostró la asociación entre la obesidad y la CVRS, se encontró que el funcionamiento físico disminuyó a medida que el IMC se aleja de los límites normales, no se detectó una relación entre la masa corporal y la depresión, la autoestima o el funcionamiento social (Swallen, Reither, Haas \& Meier, 2005). Un metaanálisis de la asociación del IMC y la CVRS, reportó que, de 42 estimaciones del efecto del IMC en la CVRS mental, solo 6 fueron estadísticamente significativas; por otro lado, de 42 estimaciones para la CVRS física, 33 fueron estadísticamente significativas y todas sugirieron que a mayor IMC menor CVRS (Ul-Haq, Mackay, Fenwick \& Pell, 2013).

Lo anterior sugiere que el aumento de IMC impacta negativamente la percepción de bienestar físico, no así del bienestar emocional; una posible explicación es que con frecuencia, las personas con sobrepeso u obesidad, se adaptan a su condición, e ignoran las áreas de su vida afectadas por el peso y el efecto negativo del IMC (Dey, Gmel \& Mohler-Kuo, 2013; Korhonen, Seppälä, Järvenpää \& Kautiainen, 2013). Además, los niveles más altos de IMC están relacionados con otras enfermedades crónicas y limitaciones funcionales, mismas que pudieran estar afectando la CVRS física (Hassan et al., 2003).

En este estudio, el ser mujer se asoció con una menor CVRS física y mental, esto es consistente con otros estudios, como el de Patiño et al.(2012) quienes sugieren que los puntajes más altos de CVRS los presentan los hombres, en la mayoría de las subescalas del SF-36. Los efectos adversos del sobrepeso y la obesidad en la salud física de mujeres, en parte se explica porque, en ellas, hay una mayor prevalencia de condiciones médicas, como limitaciones para realizar actividades diarias, síntomas respiratorios, dolor de articulaciones y padecimientos en cuello y espalda (Mond \& Baune, 2009). Por otro lado, las diferencias en CVRS mental podrían estar relacionadas con la insatisfacción con la imagen corporal, la presencia de desórdenes alimenticios (Huang, Frangakis \& Wu, 2006) y el estigma social existente hacia la obesidad (Muennig, Lubetkin, Jia, \& Franks, 2006).

Un mayor nivel de estudios se asoció con una mejor CVRS, esta tendencia es apoyada por Vilagut et al.(2008), en otros estudios la escolaridad se ha considerado como un indicador de la posición socieconómica, donde personas con mayor nivel de estudios tienen un estatus de salud mayor en comparación con aquellas cuyo nivel es bajo (Regidor et al., 1999), debido a que un estrato socieconómico menor es asociado con la reducción en los gastos familiares, cambios en los hábitos alimenticios, falta de vivienda y el aumento de la desigualdad (Rajmil, Medina-Bustos, Sanmamed, \& Mompart-Penina, 2013).

Sin embargo, en este trabajo la asociación solo se presentó para la CVRS física, esta diferencia podría ser explicada por el contraste en la percepción subjetiva de salud, considerando que las actividades diarias de cada grupo es acorde con su nivel de estudios, probablemente personas con una menor escolaridad realizan trabajos que requieren mayor esfuerzo físico, el mismo que se ve permeado por la obesidad (García-Mendizábal et al., 2009).

No se observó una asociación entre el IMC y el autocontrol, con su ausencia también se abandonó la idea de un posible efector mediador del IMC en la relación del autocontrol con la CVRS. Si bien es cierto que el autocontrol se ha relacionado con conductas que favorecen el control de peso, tales como el aumento en el consumo de frutas y vegetales, menor ingesta de alimentos altos en grasa (Gerrits et al., 2010), más participación en deportes y menos comportamiento sedentario (Wills, Isasi, Mendoza \& Ainette, 2007), es bien sabido que, el nivel de autocontrol, disminuye después de ponerlo a prueba en diferentes tareas (Muraven \& Slessareva, 2003). Además, las diferencias individuales en el autocontrol abarcan una serie de respuestas y procesos que intervienen en la regulación del comportamiento, tales como resistencia a impulsos, control de la atención, cognición, emoción y hábitos, aspectos difíciles de capturar en una sola media (Hagger et al., 2013).

Entre las limitaciones del estudio se pueden mencionar, en primer lugar, la naturaleza transversal del mismo que impide hacer consideraciones causales sobre las variables estudiadas. Si bien la literatura sobre el tema suele considerar a la CVRS como una variable dependiente, la asociación en el sentido inverso también es verosímil. Es decir, que un deterioro en la CVRS afecte la posibilidad de autocontrol e incremente el peso corporal de las personas. Una segunda limitación se relaciona con las dificultades para generalizar los datos más allá de usuarios de centros de salud como fue el caso de este trabajo. No obstante, el perfil sociodemográfico coincide en general con el de los usuarios de los centros de salud del gobierno del Distrito Federal (Instituto Nacional de Estadística y Geografía, INEGI, 2012): en su mayoría fueron mujeres, amas de casa 
o trabajadores por cuenta propia, de clase media y baja, y con un nivel educativo de bachillerato o más.

Se sugiere incluir en el análisis otras variables como la presencia de enfermedades crónicas o estilos de vida saludables, entre ellos los niveles de la actividad física que pudieran estar influyendo a la CVRS. Además, será importante incluir a personas normopeso con el fin de comparar e identificar particularidades en población con sobrepeso u obesidad y crear intervenciones enfocadas a mejorar su calidad de vida.

Los resultados sugieren que una persona con mayor autocontrol en áreas como el control de pensamientos, emociones e impulsos, además de la regulación de la conducta y ruptura de hábitos, tiene mejor percepción de bienestar en aspectos físicos y emocionales, independientemente del IMC.

\section{Referencias}

Alonso, J. (s.f.). Descripción del instrumento Cuestionario de Salud SF-12. Barcelona: IMIM-IMAS-Institut Municipal d'Investigació Médica. Recuperado de http://iryss.imim.es/iryss/PDFs/Descripcion_SF12_BiblioPRO.pdf

Baumeister, R. F., Vohs, K. D., \& Tice, D. M. (2007). The Strength Model of Self-Control. Current Directions in Psychological Science, 16(6), 351-355. doi:10.1111/j.1467-8721.2007.00534.x

Boals, A., Vandellen, M. R., \& Banks, J. B. (2011). The relationship between self-control and health: The mediating effect of avoidant coping. Psychology \& Health, 26(8), 1049-1062. doi:10.1080/08870446.2010.529139

Dey, M., Gmel, G., \& Mohler-Kuo, M. (2013). Body mass index and health-related quality of life among young Swiss men. BMC Public Health, 13(1), 1028. doi:10.1186/1471-2458-13-1028

García-Mendizábal, M. J., Carrasco, J. M., Pérez-Gómez, B., Aragonés, N., Guallar-Castillón, P., RodríguezArtalejo, F., et al. (2009). Role of educational level in the relationship between Body Mass Index (BMI) and health-related quality of life (HRQL) among rural Spanish women. BMC Public Health, 9(1), 120. doi:10.1186/1471-2458-9-120

Gerrits, J. H., O’Hara, R. E., Piko, B. F., Gibbons, F. X., Ridder, D. T. D. de., Keresztes, N., et al. (2010). Self-control, diet concerns and eater prototypes influence fatty foods consumption of adolescents in three countries. Health Education Research, 25(6), 1031-1041. doi:10.1093/her/cyq055

Hagger, M. S., Panetta, G., Leung, C.-M., Wong, G. G., Wang, J. C. K., Chan, D. K. C., et al. (2013). Chronic Inhibition, Self-Control and Eating Behavior: Test of a «Resource Depletion» Model. PLoS ONE, 8(10). doi:10.1371/journal.pone.0076888

Hassan, M. K., Joshi, A. V., Madhavan, S. S., \& Amonkar, M. M. (2003). Obesity and health-related quality of life: a cross-sectional analysis of the US population. International journal of obesity and related metabolic disorder, 27(10), 1227-1232. doi:10.1038/sj.ijo.0802396

Huang, I.-C., Frangakis, C., \& Wu, A. W. (2006). The relationship of excess body weight and healthrelated quality of life: evidence from a population study in Taiwan. International journal of obesity, 30(8), 1250-1259. doi:10.1038/sj.ijo.0803250

Instituto Nacional de Estadística y Geografía (INEGI). (2012). Mujeres y hombres en México 2011. Recuperado del archivo del Instituto Nacional de Estadística y Geografía http://inegi.org.mx

Jia, H., \& Lubetkin, E. I. (2005). The impact of obesity on health-related quality-of-life in the general adult US population. Journal of Public Health, 27(2), 156-164. doi:10.1093/pubmed/fdi025

Kearns, B., Ara, R., Young, T., \& Relton, C. (2013). Association between body mass index and healthrelated quality of life, and the impact of self-reported long-term conditions - cross-sectional study from the south Yorkshire cohort dataset. BMC Public Health, 13(1), 1009. doi:10.1186/1471-2458-13-1009

Korhonen, P. E., Seppälä, T., Järvenpää, S., \& Kautiainen, H. (2013). Body mass index and health-related quality of life in apparently healthy individuals. Quality of life research: an international journal of quality of life aspects of treatment, care and rehabilitation. doi:10.1007/s11136-013-0433-6

López-Villalta, M., \& Soto, A. (2010). Actualización en obesidad. Cuadernos de atención primaria. 17(2), 101-107.

MacKinnon, D. P. (2008). Introduction to Statistical Mediation Analysis. Nueva York: Lawrence Erlbaum.

Miller, H. V., Barnes, J. C., \& Beaver, K. M. (2011). Self-control and health outcomes in a nationally representative sample. American journal of health behavior, 35(1), 15-27. 
Mond, J. M., \& Baune, B. T. (2009). Overweight, medical comorbidity and health-related quality of life in a community sample of women and men. Obesity, 17(8), 1627-1634. doi:10.1038/oby.2009.27

Muennig, P., Lubetkin, E., Jia, H., \& Franks, P. (2006). Gender and the burden of disease attributable to obesity. American Journal of Public Health, 96(9), 1662-1668. doi:10.2105/AJPH.2005.068874

Muraven, M., \& Slessareva, E. (2003). Mechanisms of selfcontrol failure. Motivation and limited resources. Personality and Social Psychology Bulletin, 29, 894-906.

Oliva, A., Hidalgo, M., Moreno, C., Jiménez, L., Jiménez, A., Antolín, L., \& Ramos, P. (2012). Uso y riesgo de adicciones a las nuevas tecnologías entre adolescentes y jóvenes andaluces. España: Editorial Agua Clara, SL

Organización Mundial de la Salud. (2011). Obesidad y sobrepeso. Recuperado de www.who.int/mediacentre/ factsheets/fs311/es/index.html

Patiño, F. A., Aragon, E. F., Lopera, N. A., Ortiz, N. A., Pérez, E., Santamaría, J. I., \& Botero, J. (2012). Calidad de vida relacionada con la salud en usuarios de un programa de actividad física. Iatreia, 24(3), 238-249.

Rajmil, L., Medina-Bustos, A., Sanmamed, M.-J. F. de, \& Mompart-Penina, A. (2013). Impact of the economic crisis on children's health in Catalonia: a before-after approach. BMJ Open, 3(8), e003286. doi:10.1136/bmjopen-2013-003286

Regidor, E., Barrio, G., de la Fuente, L., Domingo, A., Rodriguez, C., \& Alonso, J. (1999). Association between educational level and health related quality of life in Spanish adults. Journal of Epidemiology and Community Health, 53(2), 75-82.

Renzaho, A., Wooden, M., \& Houng, B. (2010). Associations between body mass index and healthrelated quality of life among Australian adults. Quality of life research: an international journal of quality of life aspects of treatment, care and rehabilitation, 19(4), 515-520. doi:10.1007/s11136-010-9610-z

Romero-Márquez, R., \& Romero-Zepeda, H. (2010). Reflexiones sobre la calidad de vida relacionada con la salud. Revista Médica del Instituto Mexicano del Seguro Social, 48(1), 91-102.

Ruiz, M. A. (2000). Introducción a los modelos de ecuaciones estructurales. Madrid: Universidad Nacional de Educación a Distancia
Ruiz, M. A., \& Pardo, A. (2005). Calidad de vida relacionada con la salud: definición y utilización en la práctica médica. PharmacoEconomics Spanish Research Articles, 2(1), 31-43. doi:10.1007/BF03320897

Swallen, K. C., Reither, E. N., Haas, S. A., \& Meier, A. M. (2005). Overweight, Obesity, and Health-Related Quality of Life Among Adolescents: The National Longitudinal Study of Adolescent Health. Pediatrics, 115(2), 340-347. doi:10.1542/peds.2004-0678

Takahashi, Y., Sakai, M., Tokuda, Y., Takahashi, O., Ohde, S., Nakayama, T., et al. (2011). The relation between self-reported body weight and healthrelated quality of life: a cross-sectional study in Japan. Journal of Public Health (Oxford, England), 33(4), 518-526. doi:10.1093/pubmed/fdr042

Tangney, J., Baumeister, R., \& Boone, A. (2004). High Self-Control Predicts Good Adjustment, Less Pathology, Better Grades, and Interpersonal Success. Journal of Personality, 72(2), 271-322.

Torre, F., Martín-Corral, J., Callejo. A., Gómez-Vega, C., La Torre, S., Esteban, I., Vallejo, R., \& Arizaga, A. (2008). Calidad de vida relacionada con la salud y estrategias de afrontamiento ante el dolor en pacientes atendidos por una unidad de tratamiento de dolor. Revista de la Sociedad Española del Dolor, 15(2), 83-93.

Ul-Haq, Z., Mackay, D. F., Fenwick, E., \& Pell, J. P. (2013). Meta-analysis of the association between body mass index and health-related quality of life among adults, assessed by the SF-36. Obesity, 21(3), E322-327. doi:10.1002/oby.20107

Vinaccia, S., \& Quiceno, J. M. (2012). Calidad de vida relacionada con la salud y enfermedad crónica: estudios colombianos. Psychologia. Avances de la disciplina, 6(1), 123-136.

Vilagut, G., Valderas, J. M., Ferrer, M., Garin, O., LópezGarcía, E., \& Alonso, J. (2008). Interpretación de los cuestionarios de salud SF-36 y SF-12 en España: componentes físico y mental. Medicina Clínica, 130(19), 726-735.

Wills, T. A., Isasi, C. R., Mendoza, D., \& Ainette, M. G. (2007). Self-control constructs related to measures of dietary intake and physical activity in adolescents. The Journal of adolescent health: official publication of the Society for Adolescent Medicine, 41 (6), 551-558. doi:10.1016/j.jadohealth.2007.06.013 